\title{
Gestalt grouping and common onset masking
}

\author{
TODD A. KAHAN \\ University of Southern Mississippi, Hattiesburg, Mississippi \\ and \\ KATHERINE M. MATHIS \\ Millsaps College, Jackson, Mississippi
}

\begin{abstract}
A four-dot mask that surrounds and is presented simultaneously with a briefly presented target will reduce a person's ability to identity that target if the mask persists beyond target offset and attention is divided (Enns \& Di Lollo, 1997, 2000). This masking effect, referred to as common onset masking, reflects reentrant processing in the visual system and can best be explained with a theory of object substitution (Di Lollo, Enns, \& Rensink, 2000). In the present experiments, we investigated whether Gestalt grouping variables would influence the strength of common onset masking. The results indicated that (1) masking was impervious to grouping by form, similarity of color, position, luminance polarity, and common region and (2) masking increased with the number of elements in the masking display.
\end{abstract}

When attention is divided across a visual display, a person's ability to identify a briefly presented stimulus, the target, is reduced by a simultaneously presented fourdot mask that surrounds, but does not cover, the target (Enns \& Di Lollo, 1997). This masking effect is greater under divided attention conditions (Enns \& Di Lollo, 1997, Experiment 1), is insensitive to contour proximity (Enns \& Di Lollo, 1997, Experiment 2), and is strongest when the mask remains on the screen until a response is made (Di Lollo, Enns, \& Rensink, 2000; Enns \& Di Lollo, 2000; Jiang \& Chun, 2001). This effect has been called common onset masking (Di Lollo et al., 2000), four-dot masking (Enns \& Di Lollo, 1997), and object substitution masking (Jiang \& Chun, 2001). Herein, we refer to the effect as common onset masking. ${ }^{1}$ Common onset masking is quite different from other forms of masking and, as such, poses an interesting theoretical puzzle (Enns \& Di Lollo, 2000).

For example, in backward masking by pattern, a display that follows the target and whose contours spatially overlap with those of the target creates a strong masking effect. This masking effect may arise because of integration or interruption (Coltheart, 1975; Enns \& Di Lollo, 2000; Kahneman, 1968). In integration masking, the target and the mask fuse at early levels of visual processing at which contours are defined. In interruption masking, there

Portions of these data were presented at the 40th Annual Meeting of the Psychonomic Society, Los Angeles, November 1999. We are indebted to Chris Anderson, Joshua Broman-Fulks, Rachel Bru, Yolanda Crump, Leslie Frazier, Russ Gibson, Kimberly McFarland, Susan Messick, Tiffany Rayburn, John Sellinger, Chris Ward, and Tina Williams for assistance with data collection. We thank Vince Di Lollo and anonymous reviewers for their helpful comments. Please direct correspondence to either T. A. Kahan or K. M. Mathis, Department of Psychology, Bates College, Lewiston, ME 04240 (e-mail: tkahan @bates.edu or kmathis@ bates.edu). is competition for high-level brain regions involved in the recognition of objects at later levels of visual processing. Integration masking is maximal when the target-mask stimulus onset asynchrony (SOA) is $0 \mathrm{msec}$, whereas interruption masking is maximal at SOAs above 0 but trails off at longer SOAs. Explanations for integration masking rely on overlap in the target-mask contours and cannot account for common onset masking, because no overlap exists. Explanations for interruption masking, which can occur under focused attention, cannot account for common onset effects, which are maximal under divided attention conditions.

In metacontrast masking, the visibility of a briefly presented target is reduced when the target is followed by a masking pattern whose contours are in close proximity to it. Although this effect may appear to be quite similar to common onset masking, since the contours of the mask and the target do not overlap, the two effects have quite different characteristics. For example, metacontrast masking can occur under focused attention conditions, is critically dependent on temporal succession of the target and the mask, and is influenced by contour proximity. The contours of the mask must be spatially near those of the target in order for masking to be found (Breitmeyer, 1984). Common onset masking, on the other hand, is greatest under divided attention conditions, occurs when the target and the mask appear simultaneously, and is insensitive to contour proximity (Enns \& Di Lollo, 1997).

To account for common onset masking under divided attention, Enns and Di Lollo (1997; Di Lollo \& Enns, 1998; Di Lollo et al., 2000) have proposed a theory of object substitution. This theory is based on neurophysiological data that demonstrate how brain regions invariably communicate in a bidirectional manner, using reentrant pathways (e.g., Felleman \& Van Essen, 1991; Sillito, Jones, Gerstein, \& West, 1994;Zeki, 1993). When 
objects are presented in the visual field, they are coded at several levels within the information-processing system. Low-level brain regions send their output to higher level regions that represent the information more abstractly. A perceptual hypothesis regarding the objects in the visual field is developed in the higher level areas and is checked against continued activation in the lower level areas. If continued activation in lower level regions is consistent with the initial perceptual hypothesis, the developing percept is strengthened. However, if continued activation in lower level brain regions is inconsistent with the initial perceptual hypothesis, the percept will be altered.

This iterative checking procedure is responsible for common onset masking. The target and the mask have simultaneous onsets, but the mask has a delayed offset. Because of this temporal arrangement, when an initial representation of target-plus-mask is checked against lower level activation, a mismatch develops. This will occur because the lower level activation supports a perceptual hypothesis of the mask alone; the mask remains visible without the target. The iterative checking procedure results in the substitution of the target object by the blank square formed from the four dots-hence, object substitution.

This theory has been formalized in a computational model (CMOS) that accurately predicts the empirical data (see Di Lollo et al., 2000, for a detailed explanation of the CMOS model). Variables that impair target identification increase the number of iterations needed to identify the target. This enables the model to easily account for the relationship between spatial attention and common onset masking. When attention is divided, more iterations will be necessary to identify the target. If the masking pattern remains active in lower level regions, the initial hypothesis of target-plus-mask will be supplanted with the emerging hypothesis of mask alone. Similarly, target accuracy will decrease as a function of the set size in which a target is embedded. As set size increases, more iterations will be required to identify the target among the distractors, resulting in increased masking.

\section{PERCEPTUAL GROUPING AND COMMON ONSET MASKING}

The phenomenological experience of this effect is that a mask surface replaces the target. This impression is not unique to individuals in our lab. According to Neill, Hutchison, and Graves (2002), "Not only does the space inside the dots appear blank, but there is a strong subjective impression of the contours of a square connecting the dots. Furthermore, there is a subjective impression of enhanced brightness of the area within the square, very similar to the brightness enhancement that occurs within illusory contours or subjective contours" (p. 683). Di Lollo et al. (2000) have made similar statements, claiming that the four-dot mask "was entirely adequate for defining a trailing configuration (a square surface) that replaced the target as the object of perception" (p. 492).

Is common onset masking reliant upon Gestalt grouping? To date, common onset masking has been examined only under conditions in which grouping would be likely. We sought to determine whether Gestalt grouping manipulations would moderate the masking effect. We manipulated grouping by form, similarity of color, position, luminance polarity, and common region. We predicted that we would find more masking when the dots formed a cohesive group than when the dots were ungrouped. The more object-like the mask appeared, the more likely it was predicted to replace the target as the object of perception. We use the term dots as a generic term for the elements that made up the mask. The form of the dots was manipulated across experiments, as squares, partial squares, circles, or partial circles. In Experiment 1, the mask was created with four squares or four partial squares.

\section{EXPERIMENT 1}

In general, two methods have been used to calculate common onset masking. One method is to present a target along with a surrounding four-dot mask, leaving the mask on the screen. Target accuracy is compared with conditions in which the masking pattern appeared in a location different from the target (hereafter, the unmasked control method; e.g., Enns \& Di Lollo, 1997). The other method is to present the target along with a surrounding four-dot mask and manipulate the length of time the mask is left on the screen. Comparisons are made between target accuracy for briefly presented masks and that for masks that remain visible for longer durations (hereafter, the briefly masked control method; e.g., Di Lollo et al., 2000). In the experiments reported herein, we used the unmasked control method with singly presented targets (see Figure 1 for an example). ${ }^{2}$

In prior investigations, targets and masks were randomly and independently allowed to appear in one of several locations. One methodological concern that arises from using this procedure is that the probability of the target's appearing in the same location as the mask is lower than the probability of a target's appearing in a different location from the mask. If subjects can localize the masking pattern, they may adopt a strategy of searching for the target in a different location, thereby reducing accuracy in the masked condition, relative to the unmasked condition. To ensure that a strategy of this sort was not contaminating the masking effects reported here, half of the targets and masks appeared in the same location, and half appeared in a different location. ${ }^{3}$

In this experiment, we manipulated the probability that the four-dots would be seen as a cohesive object by varying the form of the mask. The elements that made up the four-dot mask were either squares or partial squares. If the four elements formed a group, more masking might be observed; if the four elements did not form a group, less masking might be observed.

\section{Method}

Subjects. Twenty-six undergraduates enrolled in psychology courses at the University of Southern Mississippi participated for extra credit. All the subjects had normal or corrected-to-no rmal vision. 
A) Masked condition

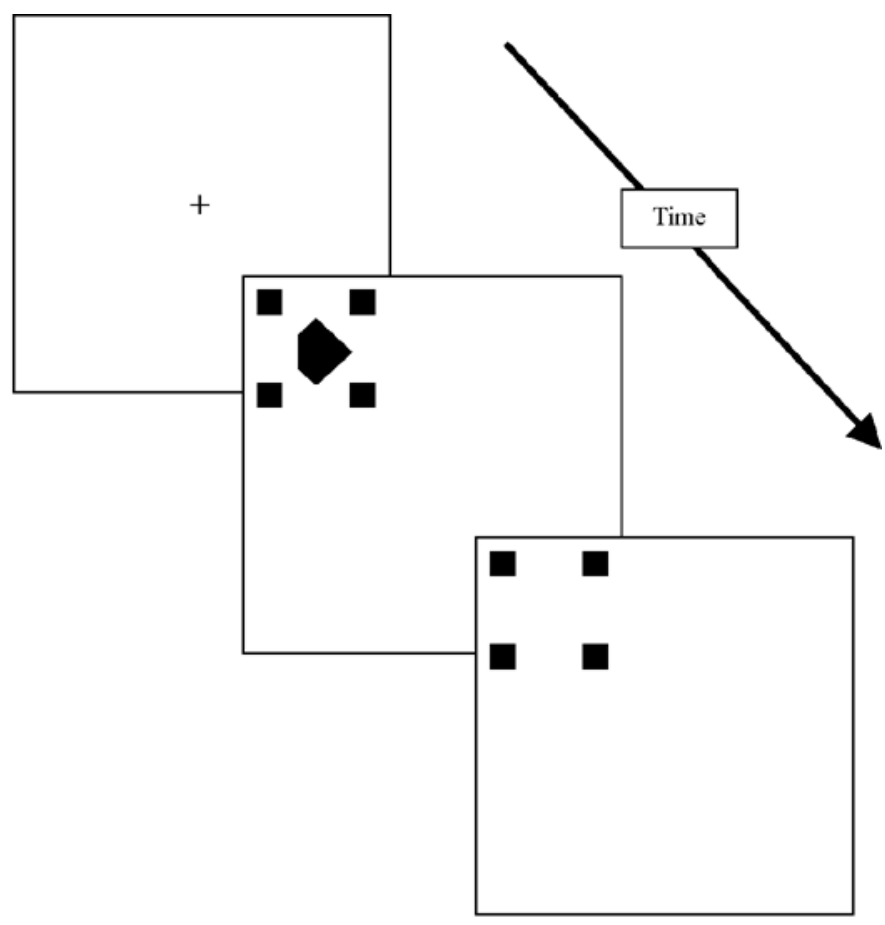

B) Unmasked condition

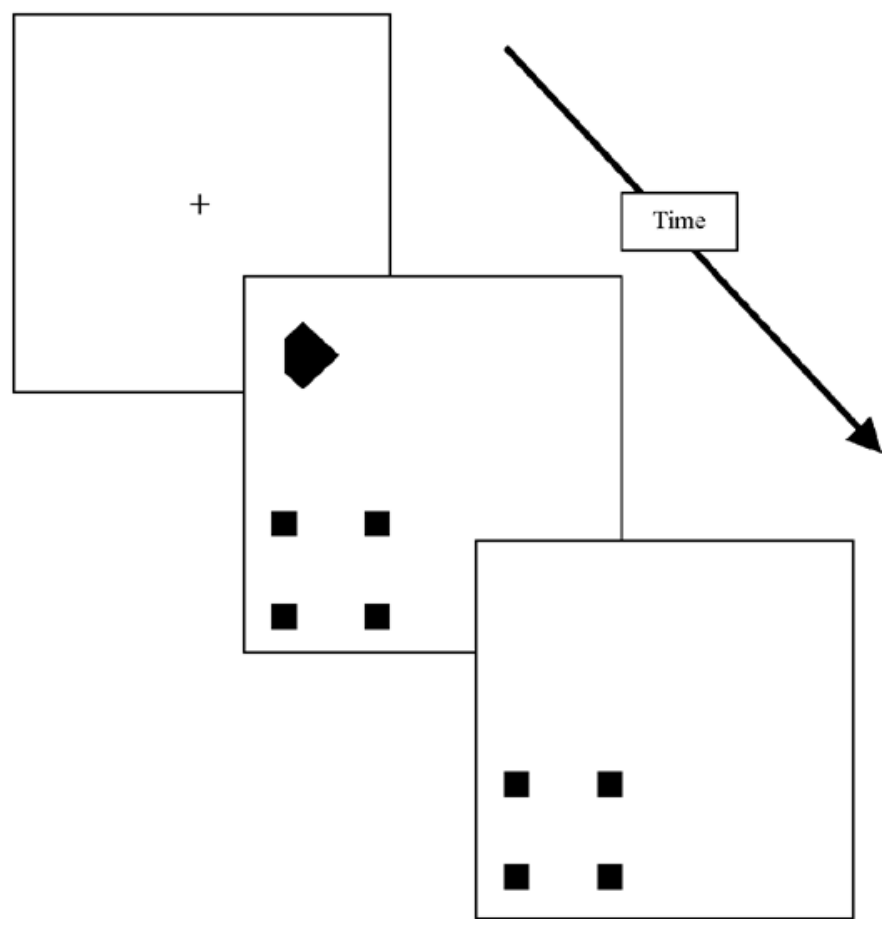

Figure 1. Example illustrating the unmasked control method in (A) masked and (B) unmasked conditions. Masking effects are calculated by computing the difference in accuracy for identifying the target in the masked and the unmasked conditions. The target and distractors were displayed in white on a black background.

Materials and Procedure. Each trial began with a fixation point $(+)$ centered on the computer screen for $250 \mathrm{msec}$. Following fixation, the target and the mask appeared simultaneously. On each trial, the subjects decided which of two possible targets had been presented for $33 \mathrm{msec}$ on a black screen: a white diamond $\left(1.24^{\circ}\right.$ in vertical extent) with the corner $\left(0.34^{\circ}\right)$ missing on either the left- or the right-hand side. The subjects were instructed to press the " $Z$ " key on the keyboard with their left index fingers if the corner was 


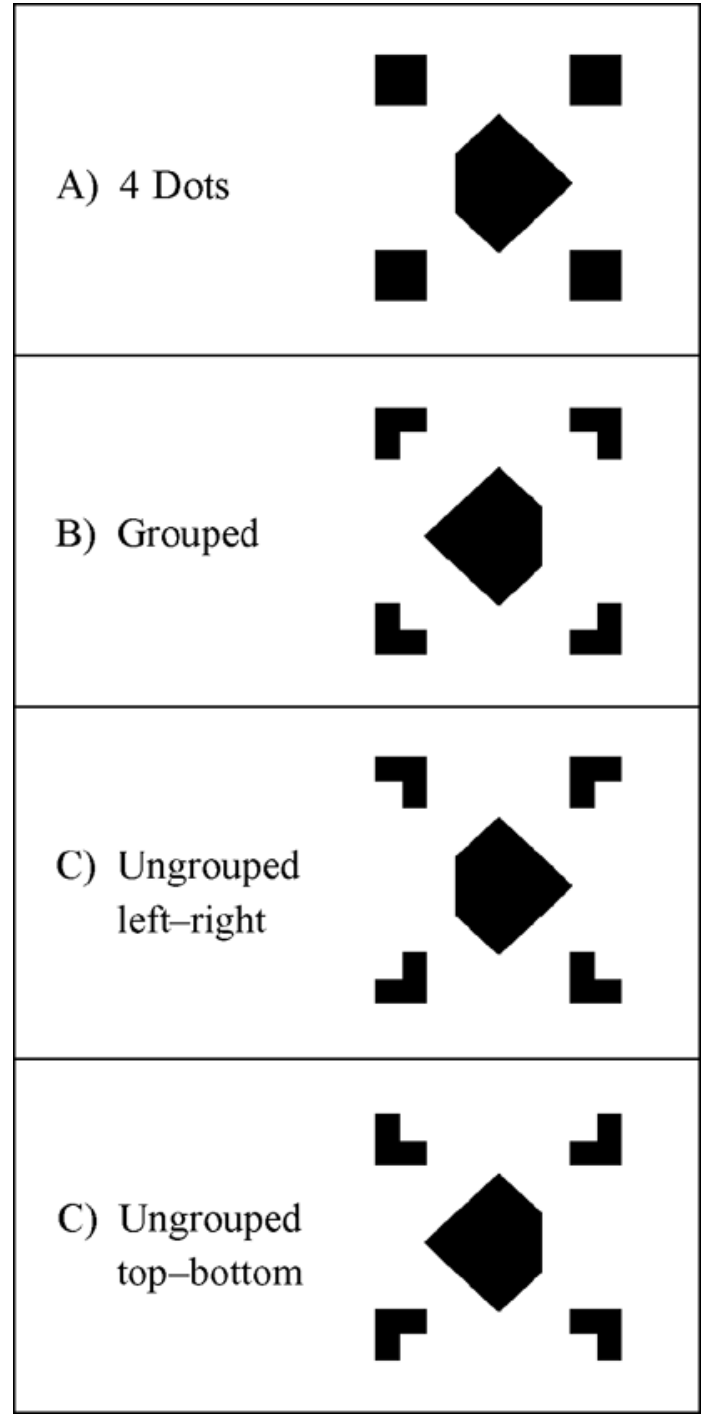

Figure 2. Masking patterns used in Experiment 1 along with an example target missing either the leftmost ( $A$ and $C$ ) or the rightmost (B and D) corner. (A) Four-dot, (B) grouped, (C) ungrouped left-right, and (D) ungrouped top-bottom masks are shown. The target and distractors were displayed in white on a black background.

missing from the left side of the diamond and the "/?" key with their right index fingers if the corner was missing from the right. This target randomly appeared in one of four quadrants on the computer screen $\left(4.30^{\circ}\right.$ from center). Each target was presented along with a masking pattern of four dots $\left(0.40^{\circ}\right)$. These dots were arranged in an apparent square $\left(2^{\circ}\right.$ on a side), sometimes surrounding the target, other times appearing in a different quadrant (see Figure 2A). When the dots surrounded the target, the closest distance between the target and the mask contours was $0.70^{\circ}$, far too distant for effective masking by contour (Breitmeyer, 1984). A small corner $\left(0.2^{\circ}\right)$ was removed from each square dot $75 \%$ of the time, creating partial square dots (see Figures 2B-2D). Each of the masking patterns depicted in Figure 2 was displayed $25 \%$ of the time. These patterns randomly appeared in one of the four quadrants and were shown in the same location as the target half of the time. After
$33 \mathrm{msec}$, the target disappeared, and the masking pattern remained on the screen until a response was made. The program was created using Micro Experimental Laboratory (MEL v2.01) software (Schneider, 1988) and was presented on an IBM-compatible PC.

\section{Results}

The proportion of correct responses was computed as a function of whether the masking pattern appeared in a location that was the same as (masked) or different from (unmasked) that of the target and whether a four-dot, grouped, or ungrouped pattern was used. No differences in the data were found between the two ungrouped patterns, left-right and top-bottom, so the data have been collapsed across these groupings. These data are depicted in Table 1 . The data were then submitted to a $3 \times 2$ analysis of variance (ANOVA), with grouping (four-dot vs. grouped vs. ungrouped) and masking (masked vs. unmasked) as within-subjects variables. A main effect of masking $\left[F(1,25)=90.15, M S_{\mathrm{e}}=0.01\right]$ indicated that, overall, the subjects performed worse in the masked condition than in the unmasked condition. No main effect of grouping or interaction between masking and grouping was obtained. The grouping manipulations made here were ineffective in altering masking.

\section{EXPERIMENT 2}

The results of Experiment 1 did not indicate an effect of grouping by form. In Experiment 2, we manipulated grouping by similarity of color. We also examined masking from eight dots, because four dots that remain on the screen may always be grouped together. The dots were presented in either a $2 \times 2$ or a $4 \times 2$ array (rows $\times$ columns, yielding four- and eight-element masks, respectively). The $2 \times 2$ arrangement was identical to that in Experiment 1. Adding one row above and below the central two rows created the $4 \times 2$ arrangement. In either case, either the central four dots that surrounded the target were the same color (grouped), or two were the same color and two were different (ungrouped). When eight dots were used, they were colored so that they would either group across the target object (central dots, same color; peripheral dots, different) or to either side (top dots, same color; bottom dots, different).

\section{Method}

Subjects. Sixty undergraduates enrolled in psychology courses at the University of Southern Mississippi participated for extra credit. One half viewed four-dot masks, and the other half viewed eight-dot masks. All the subjects had normal color vision and normal or corrected-to-normal visual acuity.

Materials and Procedure. The procedure was similar to that used in Experiment 1. One half of the subjects viewed a four-dot mask (Figure 3A); the others viewed an eight-dot mask (Figure 3B). When four dots were used, the dots were either all the same color (red or green) (grouped; Figure 3Ai) or two were the same (red) while the other two were different (green) (ungrouped; Figure 3Aii). When the dots were ungrouped, either the top two were different from the bottom two, or the left two were different from the right two. The elements that made up the four-dot mask were squares; 
Table 1

Experiment 1 Data:

Mean Proportion of Correct Target Identification as a Function of Masking and Grouping Conditions

\begin{tabular}{llcc}
\hline Condition & 4 Dots & Grouped & Ungrouped \\
\hline Unmasked & .83 & .84 & .83 \\
Masked & .69 & .68 & .66 \\
Masking & $.14^{*}=$ & $.16^{*}=$ & $.17^{*}$ \\
\hline
\end{tabular}

Note-Grouping was manipulated by varying the form of the masking pattern. $* p<.05$.

the elements that made up the eight-dot masks were partial circles. The squares and partial circles subtended the same visual angle $\left(0.40^{\circ}\right)$. When eight dots were used, grouping was made stronger by manipulating color and form simultaneously. The eight dots could either encompass the target (grouped) or not (ungrouped), creating Kanizsa (1976) squares. When the partial circles were grouped, the four inner dots had segments missing, so that the missing portion formed a square. These inner four dots were all the same color (either red or green), whereas the upper and lower dots were displayed in different colors and were arranged so that grouping with the center four dots would be unlikely (see Figure 3Bi). When the partial circles were ungrouped, the top four and bottom four dots each had segments missing, so that the missing portions formed two squares, one above and one below the target location. The top four and bottom four dots were displayed in different colors (see Figure 3Bii). The dots were grouped half of the time and ungrouped half of the time.

\section{Results}

The proportion of correct responses was computed as a function of whether the mask appeared in a location that was the same as or different from that of the target, whether the patterns were grouped or ungrouped, and whether the masking pattern was composed of four dots or eight dots. Because masking was equivalent for red versus green and left-right versus top-bottom masks, the data were averaged across these variables. The data are depicted in Table 2 and were submitted to a $2 \times 2 \times 2$ ANOVA, with grouping (grouped vs. ungrouped) and masking (masked vs. unmasked) as within-subjects variables and number of dots (four vs. eight) as a betweensubjects variable. A main effect of masking $[F(1,58)=$ $\left.184.14, M S_{\mathrm{e}}=0.009\right]$ indicated that, overall, the subjects performed worse in the masked condition than in the unmasked condition. A main effect of number of dots $\left[F(1,58)=14.40, M S_{\mathrm{e}}=0.004\right]$ indicated that, overall, the subjects performed worse in the eight-dot condition than in the four-dot condition. No main effect of grouping or interaction between masking and grouping was obtained. An interaction between masking and number of dots indicated that eight dots produced more masking $(20 \%)$ than did four dots $\left[13 \% ; F(1,58)=8.12, M S_{\mathrm{e}}=\right.$ 0.009 ; Fisher's LSD $=5 \%$ ]

Because number of dots in the mask (four vs. eight) was confounded with type of dot (square vs. partial circle) and manner of grouping (color alone vs. color and form together), firm conclusions are difficult to reach. One possibility is that masking increases as the number of elements in the display increases (cf., Jiang \& Chun,
2001, Experiment 5). According to object substitution theory, as the number of iterations increases, so too will masking. If attention is deployed to the target more slowly, as the number of elements in the display increases, the number of iterations required to identify the target will increase, resulting in greater masking. This

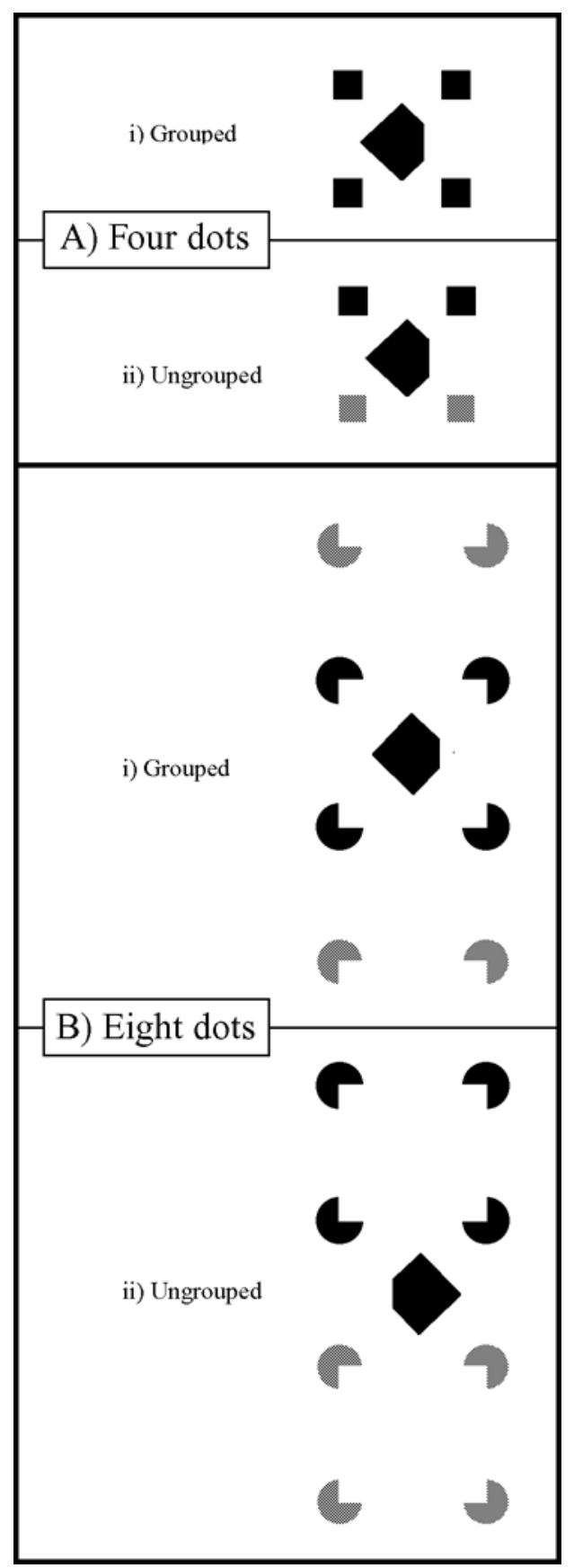

Figure 3. Example (A) four-dot and (B) eight-dot masks in (i) grouped and (ii) ungrouped patterns, used in Experiment 2, along with example target shapes. The dots were shown in either red or green (depicted here with shading). The background was black, and the target was white. 
Table 2

Experiment 2 Data:

Mean Proportion of Correct Target Identification as a

Function of Masking, Grouping, and Number of Dots in the Masking Display

Number of Dots in Masking Display

\begin{tabular}{|c|c|c|c|c|}
\hline \multirow[b]{3}{*}{ Condition } & \\
\hline & \multicolumn{2}{|c|}{ Four Dots } & \multicolumn{2}{|c|}{ Eight Dots } \\
\hline & Grouped & Ungrouped & Grouped & Ungrouped \\
\hline Unmasked & .81 & .81 & .76 & .74 \\
\hline Masked & .68 & .68 & .55 & .55 \\
\hline Masking & $.13^{*}$ & $.13^{*}$ & $.21 *$ & $.19 *$ \\
\hline
\end{tabular}

Note-Grouping was manipulated by varying the color (four and eight dots) and form (eight dots) of the masking pattern. $* p<.05$.

possibility was explored in Experiment 3 . Two dots were used, rather than four or eight. If the number of elements in a display influences masking, reduced masking should be obtained with two dots. Regardless, the data from Experiment 2 demonstrated that masking was unaltered when dots were grouped by similarity of color.

\section{EXPERIMENT 3}

In Experiments 1 and 2, we manipulated the likelihood with which a masking pattern would be perceived as a cohesive object by manipulating form and color. Despite these manipulations, masking was influenced only by number of elements in the display. It is possible that our grouping manipulations were not strong enough.

In Experiment 3, we used a two-dot mask and manipulated whether the dots crossed the target location. If two dots delineate a line, one might expect greater masking when the dots "cross" the spatial location of the target than when the dots are spatially adjacent to the target. In the former, the masking "object" appears in the same spatial location and may supplant the target's percept; in the latter, the masking "object" appears adjacent to the target and should not substitute for the target. In addition, in this experiment, we tested the supposition that masking increases with the number of dots in the masking display. Because Experiment 2 confounded number of dots in the mask (four vs. eight) with type of dot (square vs. partial circle) and manner of grouping (color alone vs. color and form together), firm conclusions were difficult to reach. In the present experiment, two-dot masking was examined while the confounding variables were held constant. The dots were all white squares; form and color were not manipulated.

In this experiment, we sought to determine whether (1) spatial arrangement and (2) number of elements in the display would influence common onset masking. We predicted less masking for dots adjacent to the target than for dots crossing the target and less masking for two-dot displays than for four-dot displays.

\section{Method}

Subjects. Undergraduates enrolled in psychology courses at the University of Southern Mississippi participated for extra credit. All the subjects had normal or corrected-to-normal vision. Since we wanted to determine whether masking would vary depending on whether the dots crossed the spatial location of the target and since we reasoned that this interaction, if there, might be small, a large sample was gathered. A total of 75 subjects participated.

Materials and Procedure. In terms of timing, the methods were identical to those used previously. However, only two dots appeared as the masking pattern in this experiment. These dots could appear in the upper, lower, leftmost, rightmost, or crossed (left to right or right to left) positions (see Figure 4). The arrangement of the dots was determined randomly. If the dots were arranged so that they would cross the spatial location of the target, they were coded as grouped (see Figure 4A). If the dots appeared in the upper, lower, leftmost, or rightmost positions, they were coded as ungrouped, since these arrangements would never overlap the spatial location of the target (see Figure 4B).

\section{Results}

The proportion of correct responses was computed as a function of whether the masking pattern appeared in a quadrant that was the same as (masked) or different from (unmasked) quadrant that of the target and whether the pattern remained adjacent to or crossed the target location. The data are depicted in Table 3 and were submitted to a $2 \times 2$ ANOVA, with grouping (adjacent vs. crossed arrangements) and masking (masked vs. unmasked) as within-subjects variables. The only effect that reached significance was a main effect of masking $[F(1,74)=$ $\left.48.749, M S_{\mathrm{e}}=0.006\right]$. The grouping variable (adjacent vs. crossed) did not influence masking. Furthermore, the twodot effects reported herein appear to have been smaller than the four-dot effects reported in Experiments 1 and 2; these, in turn, appear to have been smaller than the eightdot effect reported in Experiment 2 (see Table 4). When masking under divided attention was examined across all three experiments, collapsing across grouping variables, more masking was found for eight dots (20\%), followed by four dots (14\%) and two dots (6\%). This was supported with an interaction between number of dots (two, four, or eight) and masking [masked vs. unmasked; $F(2,158)=$ $\left.30.99 ; M S_{\mathrm{e}}=0.004\right]$.

\section{EXPERIMENT 4}

In Experiment 4, we manipulated grouping by luminance polarity. We chose luminance polarity because data support the existence of two grouping mechanisms, edge based and brightness based (Gilchrist, Humphreys, Rid-

Table 3

Experiment 3 Data:

Mean Proportion of Correct Target Identification as a Function of Masking and Grouping Condition

\begin{tabular}{lcc}
\hline Condition & Grouped & Ungrouped \\
\hline Unmasked & .78 & .80 \\
Masked & .72 & .73 \\
Masking effect & $.06^{*}$ & $=$ \\
\hline
\end{tabular}

Note-All masking patterns contained two dots. Grouping was manipulated by varying whether the two-dot masking pattern crossed the target location. $* p<.05$. 


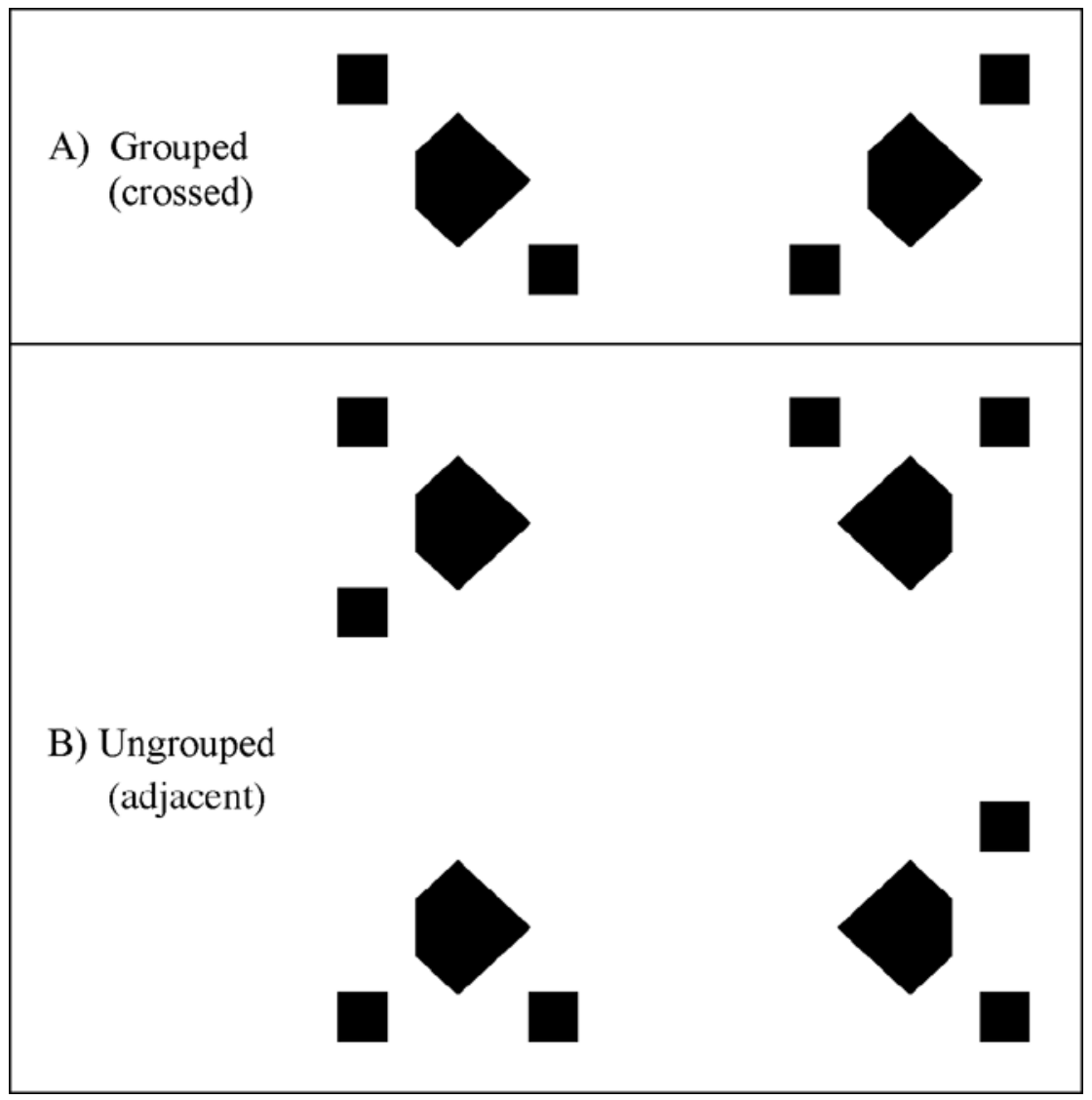

Figure 4. Masking patterns used in Experiment 3, along with example target shapes, in (A) example grouped patterns and (B) example ungrouped patterns. The target and distractors were displayed in white on a black background.

doch, \& Neumann, 1997; cf., Grossberg \& Mingolla, 1985; Grossberg \& Todorović, 1988). The brightness system is sensitive to luminance polarity manipulations; the edge system is not. Gilchrist et al. found that a person's ability to group dots is influenced by luminance polarity when edge information does not obscure it. In their experiments, subjects were required to search a display for a target. The target was a vertically aligned pair of dots, and the distractors were horizontally aligned dots. Display size and luminance polarity were manipulated. In order to locate the target, the subjects had to group the dots. Search times for same-polarity targets remained relatively flat across display size but increased linearly when reversepolarity dots were used. Slopes for same-polarity light circles were shallower than those for same-polarity dark circles, suggesting that darker circles were more difficult to group. Slopes for reverse-polarity circles were the greatest, suggesting that reverse-polarity circles are not grouped automatically. When collinear edges could be used to group the target, reverse polarity had little effect.

In the present experiment, circles, rather than squares, were used to eliminate grouping based on edge informa-

Table 4

Experiments 1-3: Masking as a Function of the Number of Dots in the Masking Display, Collapsing Across Grouping Variables

\begin{tabular}{|c|c|c|c|}
\hline \multirow[b]{2}{*}{ Condition } & \multicolumn{3}{|c|}{ Number of Dots in the Masking Display } \\
\hline & $\begin{array}{c}\text { Two } \\
\text { (Experiment 3) } \\
\end{array}$ & $\begin{array}{c}\text { Four } \\
\text { (Experiments } 1 \text { and 2) }\end{array}$ & $\begin{array}{c}\text { Eight } \\
\text { (Experiment 2) }\end{array}$ \\
\hline Unmasked & .79 & .82 & .75 \\
\hline Masked & .73 & .68 & .55 \\
\hline Masking effect & $.06^{*}$ & $.14 *$ & $.20 *$ \\
\hline
\end{tabular}




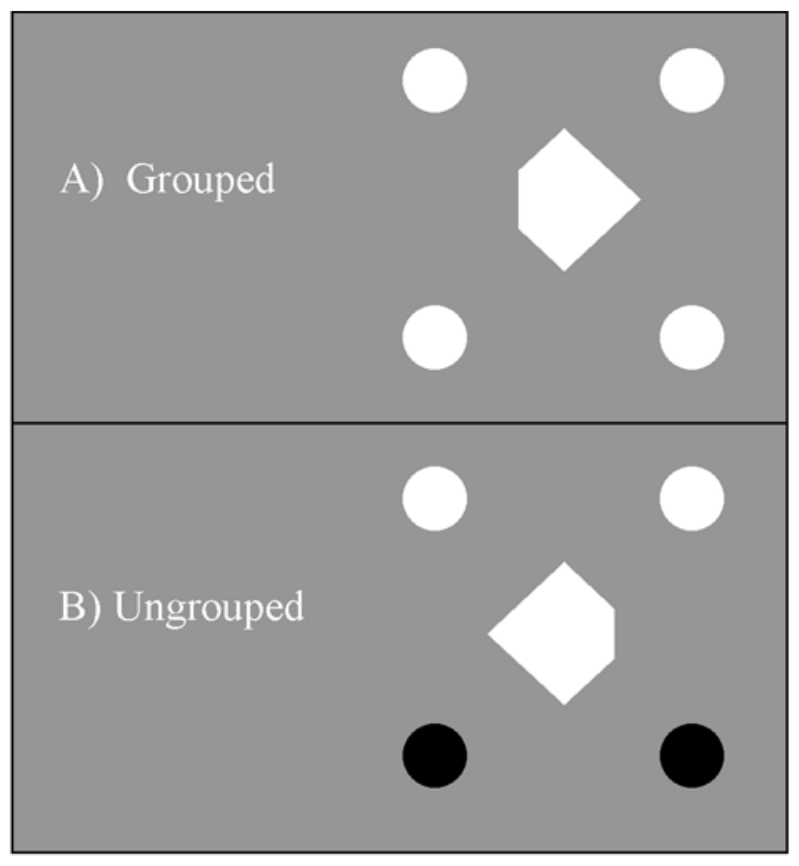

Figure 5. Masking patterns used in Experiment 4, along with example target shapes, in (A) example grouped patterns and (B) example ungrouped patterns.

tion. The four circles were either the same polarity (grouped) or reverse polarity (ungrouped). Same-polarity masking patterns were composed of four circles that were lighter than the background. Light circles were used because they could be grouped more easily than dark circles (Gilchrist et al., 1997, Experiment 2). Reverse-polarity masking patterns were composed of two lighter-thanbackground and two darker-than-background circles located in the top and the bottom positions of the square, respectively. If common onset masking is sensitive to brightness-based grouping manipulations, more masking should be obtained when the dots are easily grouped (same polarity) than when the dots are more difficult to group (reverse polarity).

\section{Method}

Subjects. Twenty-five undergraduates enrolled in psychology courses at the University of Southern Mississippi participated for extra credit. All the subjects had normal or corrected-to-normal visual acuity.

Materials and Procedure. The procedure was similar to that in Experiment 1, with the exception that circles, rather than squares, were used and grouping was manipulated by luminance polarity, rather than by form. The diameter of the circles was the same as the width of the squares. When the circles were grouped, all four were lighter than the background (see Figure 5A). When the circles were ungrouped, the top two were lighter than the background, and the bottom two were darker than the background (see Figure 5B). The circles were grouped half of the time and ungrouped half of the time.

\section{Results}

The proportion of correct responses was computed as a function of whether the mask appeared in a location that was the same as or different from that of the target and whether the patterns were grouped or ungrouped. The data are depicted in Table 5 and were submitted to a $2 \times 2$ ANOVA, with grouping (grouped vs. ungrouped) and masking (masked vs. unmasked) as within-subjects variables. A main effect of masking $[F(1,24)=24.9$, $\left.M S_{\mathrm{e}}=0.01\right]$ indicated that, overall, the subjects performed worse in the masked than in the unmasked condition. No main effect of grouping or interaction between masking and grouping was obtained. These data demonstrate that common onset masking was unaltered when circles were grouped by luminance polarity.

In Experiments 1 and 2, when the background was black, four elements produced $14 \%$ masking. When the background luminance was increased, masking decreased to $10 \%$. More masking was obtained in Experiments 1 and 2 than in Experiment 3; comparing these experiments, there was an interaction between masking and background luminance $\left[F(1,79)=4.35, M S_{\mathrm{e}}=0.004\right]$. Possible explanations for this effect will be addressed in the General Discussion section.

\section{EXPERIMENT 5}

In Experiment 5, we manipulated grouping by common region. Grouping by common region is a strong and unique Gestalt principle that can overcome grouping by proximity and similarity (Palmer, 1992). Because of the strength of this effect and its ability to overcome other powerful grouping manipulations, we reasoned that grouping by common region might moderate common onset masking. An example of this grouping principle is depicted in Figure 6A, in which the six squares are seen as forming three separate groups. In Figures $6 \mathrm{~B}$ and $6 \mathrm{C}$, the squares are still seen as three groups, demonstrating that common region can overcome grouping by similarity of color and proximity, respectively. If the masking elements belong to a common region that surrounds the target, more masking may be obtained, relative to elements in different regions adjacent to the target.

Because masking increases with the number of elements in the display, we hypothesized that the addition of a common region pattern might also increase masking. If the addition of elements to the display slows the rate by which attention can be deployed to the target, more masking should be found relative to the four element displays used in Experiments 1 and 2.

Table 5

Experiment 4 Data:

Mean Proportion of Correct Target Identification as a Function of Masking and Grouping Conditions

\begin{tabular}{lcc}
\hline Condition & Grouped & Ungrouped \\
\hline Unmasked & .83 & .83 \\
Masked & .73 & .72 \\
Masking effect & $.10^{*}$ & $=$ \\
\hline
\end{tabular}

Note-Grouping was manipulated by varying luminance polarity. ${ }^{*} p<$ .05 . 
A)

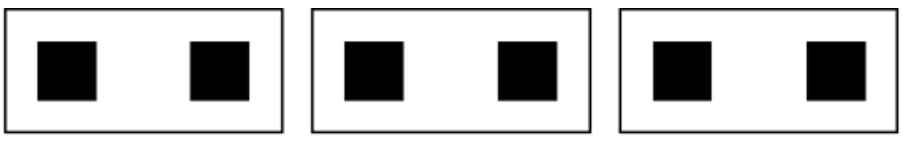

B)

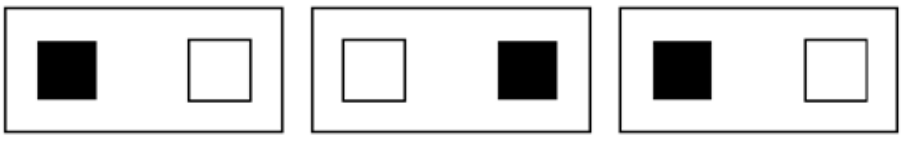

C)
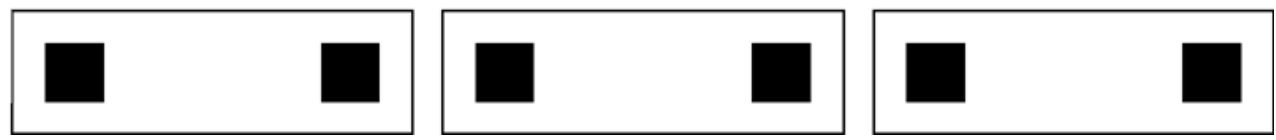

Figure 6. Examples of (A) grouping by common region, (B) common region overcoming grouping by similarity, and $(C)$ common region overcoming grouping by proximity.

\section{Method}

Subjects. Twenty-four undergraduates enrolled in psychology courses at the University of Southern Mississippi participated for extra credit. All the subjects had normal or corrected-to-normal visual acuity.

Materials and Procedure. The procedure was similar to that in Experiment 1, with the exception that grouping was manipulated by common region, rather than by form. When the elements of the mask (always squares in this experiment) were grouped, they were contained within a larger square region (see Figure 7A). When the elements of the mask were ungrouped, they were contained within different rectangular regions; the top two were separated from the bottom two (see Figure 7B). The masks were grouped half of the time and ungrouped half of the time.

\section{Results}

The proportion of correct responses was computed as a function of whether the mask appeared in the same or a different location from the target and whether the patterns were grouped or ungrouped. The data are depicted in Table 6 and were submitted to a $2 \times 2$ ANOVA, with grouping (grouped vs. ungrouped) and masking (masked vs. unmasked) as within-subjects variables. A main effect of masking $\left[F(1,23)=61.03, M S_{\mathrm{e}}=0.01\right]$ indicated that, overall, the subjects performed worse in the masked than in the unmasked condition. No main effect of grouping or interaction between masking and grouping was obtained. These data demonstrate that common onset masking was unaltered when squares were grouped by common region.

Although grouping by common region did not moderate masking, the effects reported here $(20 \%)$ were larger than those reported in the four-dot displays of Experiments 1 and $2\left[F(1,78)=5.02, M S_{\mathrm{e}}=0.005\right]$. This is consistent with object substitution theory. The addition of elements to the display presumably decreases the rate by which attention can be deployed to the target location, increasing the number of iterations needed to identify the target and, thereby, increasing masking.

\section{GENERAL DISCUSSION}

Common onset masking was impervious to Gestalt grouping by form, similarity of color, location, lumi- nance polarity, and common region. However, common onset masking was sensitive to both background luminance and the number of elements in the masking display. Possible reasons for these effects will be discussed below.

\section{Why No Effect of Grouping?}

At least three possibilities exist as to why there was no effect of grouping. First, divided attention conditions may have precluded Gestalt grouping. This alternative,

\section{A) Grouped}
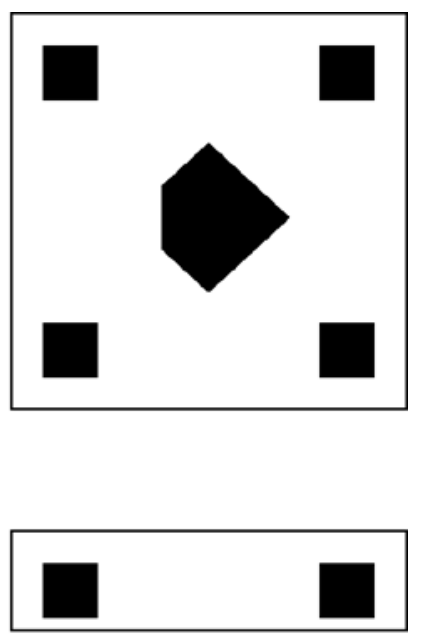

\section{B) Ungrouped}

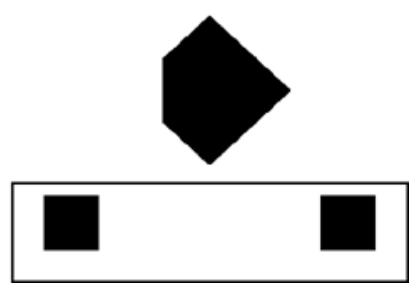

Figure 7. Masking patterns used in Experiment 5, along with example target shapes, in (A) example grouped patterns and (B) example ungrouped patterns. The target, distractors, and common region boxes were displayed in white on a black background. 
Table 6

Experiment 5 Data:

Mean Proportion of Correct Target Identification as a Function of Masking and Grouping Conditions

\begin{tabular}{ccc}
\hline Condition & Grouped & Ungrouped \\
\hline Unmasked & .75 & .74 \\
Masked & .56 & .54 \\
Masking effect & $.19 * \quad=$ & $.20 *$ \\
\hline Note-Grouping was manipulated by varying common region. $* p<.05$.
\end{tabular}

however, does not seem likely. Although data exist that indicate that subjects cannot identify Gestalt grouping patterns presented under conditions of inattention (Mack, Benyu, Tuma, Kahn, \& Rock, 1992; Moore \& Egeth, 1997), other data indicate that subjects can perceive grouping patterns presented under conditions of divided attention (e.g., Bravo \& Blake, 1990). Inattention refers to conditions in which stimuli are presented but receive no attention and subjects are unaware of their presence (e.g., the stimuli were presented in the background). Divided attention refers to conditions in which stimuli are presented and subjects are aware of their presence (e.g., the stimuli were presented in a visual search task). The terms inattention and divided attention have been used inconsistently and are presented here as Mack et al. and Moore and Egeth have used them. The conditions in the present experiments most closely resemble divided attention, for which perceptual grouping does occur (Bravo \& Blake, 1990). Furthermore, Moore and Egeth contended that Gestalt grouping also occurs under the more rigorous conditions of inattention, contrary to the conclusions of Mack et al. In their experiments, dots were presented in the background that, if grouped, would form illusions (e.g., the Ponzo illusion), and subjects were asked to guess the lengths of lines in the foreground. The results indicated that although these grouping patterns were not available for recall, they did influence the subjects' estimates of line length. These data strongly indicate that even under conditions of inattention, Gestalt grouping does occur.

A second alternative is that our grouping manipulations were simply too weak to produce an effect. For example, manipulating color to create a mixed-contrast Kanizsa square does not eliminate the perception of an illusory surface (Grove, Grossberg, \& Mingolla, 1995). However, in the present experiments, we did not manipulate grouping solely by similarity of color. We also manipulated grouping by form, location, luminance polarity, and common region. None of the grouping manipulations had an influence on masking.

The third, and most likely, alternative is that common onset masking is impervious to Gestalt grouping. Although prior experiments have always used a mask that delineated a square surface, the common onset masking effect is not dependent on this. When the Gestalt group was disrupted, masking remained stable. We have also collected data in our laboratory, using a masking pattern that forms a face. (Two eyes and a mouth were presented on a white background; the methods were essentially identical to those reported herein.) The masking face was presented either upright or upside-down; data indicate that orientation influences face recognition (Yin, 1969). Masking was unaltered by the orientation. Taken together, all of these data suggest that it is not necessary for the mask to form a cohesive unit.

\section{Why an Effect of Background Luminance?}

In Experiment 4, grouping by luminance polarity was examined. An unexpected outcome was that common onset masking decreased with increased background luminance; masking from four dots was greater when the

A) Same side

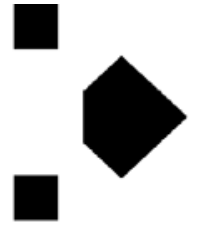

True stimulus

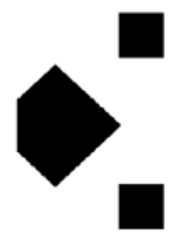

True stimulus

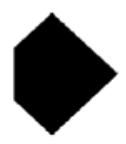

Internal representation

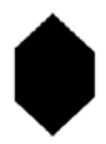

Internal representation

Figure 8. Examples of the true stimulus and the possible internal representations that could remain after a two-dot mask appears on (A) the same side or (B) the opposite side of the missing corner of the target. 
Table 7

Experiment 3 Data:

Mean Proportion of Correct Target Identification When

Two-Dot Masks That Appeared on Either the Left or the Right

Were Used, as a Function of Whether the Mask Was on the Same or the Opposite Side of the Missing Corner

\begin{tabular}{lcc}
\hline & \multicolumn{2}{c}{ Corner-Mask Relationship } \\
\cline { 2 - 3 } Condition & Same Side & Different Side \\
\hline Unmasked & .81 & .77 \\
Masked & .79 & .69 \\
Masking effect & $.02^{\dagger}<$ & $.08^{*}$ \\
\hline
\end{tabular}

$* p<.05 . \quad \dagger p>.05$.

background was black (Experiments 1 and 2) than when the background luminance increased. Greater luminance levels alone cannot explain the reduced masking effects. This can be ruled out because the addition of elements to the display, which increased luminance levels, produced greater masking (Experiment 2).

One possibility for this effect is that common onset masking is sensitive to manipulationsin mask-background contrast. This explanation seems reasonable if one imagines the extreme case in which the masking dots and the background are virtually indistinguishable. If the maskbackground contrast is too low, the visual system may discard the mask as a possible object for substitution. This possibility remains to be empirically tested.

\section{Why an Effect of the Number of Dots?}

The number of elements in the masking display is positively correlated with the magnitude of masking. The data indicate that common onset masking results from reentrant processing in the visual system and that variables that increase the number of iterations needed to identify the target increase masking (Enns \& Di Lollo, 2000). If the total time for attention to make contact with the target ( $t_{c}$ in CMOS; see Di Lollo et al., 2000) increases with the number of elements in the display, the number of iterations necessary to identify the target will increase. For this reason, these data are consistent with a theory of object substitution (Di Lollo et al., 2000).

To find converging evidence for the object substitution hypothesis, we conducted a post hoc analysis of the Experiment 3 data. In Experiment 3, two dots adjacent to the target produced reliable masking. If the mask supplants nearby information, more masking may be found when the mask appears on the opposite side from the missing corner than when the mask appears on the same side as the missing corner. Losing the intact corner should hurt performance more than does losing the side without the corner, because the intact corner is more informative (it informs the subject that the missing corner was on the opposite side). This has been illustrated in Figure 8. If the mask is presented on the same side as the missing corner, subjects will be left with the internal representation depicted in Figure 8A. However, if the mask is presented on the opposite side, subjects will be left with the internal representation depicted in Figure 8B. If this is correct, subjects should have superior performance when the masking pattern and missing corner appear on the same side, rather than on different sides. Two-dot masks appearing on the left or the right were examined as a function of whether they appeared on the same or the opposite side of the missing corner and whether they appeared in a quadrant that was the same as or different from that of the target. The hypothesis was supported (see Table 7). The statistical interaction depicted in Table 7 was reliable $\left[F(1,74)=4.025, M S_{\mathrm{e}}=\right.$ 0.017]. A Fisher's least significant difference of .04 $(p<$ .05 ) indicates that significant masking was obtained when masks appeared on the opposite side of the missing corner, but not when masks appeared on the same side. Although this post hoc analysis is consistent with the object substitution account described, it is possible that these results are merely a consequence of response bias. If subjects are uncertain, they may have a bias to respond left when the masking pattern is on the left and right when the masking pattern is on the right. If such a bias exists, no masking should be found when the mask and the target appear on the same side. When the mask and the target appear on the same side, people will do well because of this bias. When the mask and the target appear on opposite sides, the bias will no longer be beneficial. ${ }^{4}$

In summary, these data afford several conclusions. First, common onset masking does not appear to be influenced by Gestalt grouping based on form, similarity of color, position, luminance polarity, or common region. Second, the magnitude of common onset masking is positively related to the numbers of elements in the display. These results are broadly consistent with a theory of object substitution in which the object need not form a meaningful Gestalt.

\section{REFERENCES}

Bravo, M., \& Blake, R. (1990). Preattentive vision and perceptual groups. Perception, 19, 515-522.

Breitmeyer, B. G. (1984). Visual masking: An integrative approach. New York: Oxford University Press.

Coltheart, M. (1975). Iconic memory: A reply to Professor Holding. Memory \& Cognition, 3, 42-48.

Di Lollo, V., \& EnNs, J. T. (1998, November). Perceiving with and without attention: Competition for consciousness among visual events. Paper presented at the 39th Annual Meeting of the Psychonomic Society, Dallas.

Di Lollo, V., EnNs, J. T., \& Rensink, R. A. (2000). Competition for consciousness among visual events: The psychophysics of reentrant visual processes. Journal of Experimental Psychology: General, 129, 481-507.

ENNS, J. T., \& Di Lollo, V. (1997). Object substitution: A new form of masking in unattended visual locations. Psychological Science, $\mathbf{8}$, 135-139.

Enns, J. T., \& Di Lollo, V. (2000). What's new in visual masking? Trends in Cognitive Sciences, 4, 345-352.

Felleman, D. J., \& VAn Essen, D. C. (1991). Distributed hierarchical processing in primate visual cortex. Cerebral Cortex, 1, 1-47.

Gilchrist, I. D., Humphreys, G. W., Riddoch, M. J., \& Neumann, H. (1997). Luminance and edge information in grouping: A study using visual search. Journal of Experimental Psychology: Human Perception \& Performance, 23, 464-480.

Grossberg, S., \& Mingolla, E. (1985). Neural dynamics of perceptual grouping: Texture, boundaries, and emergent segmentations. Perception \& Psychophysics, 38, 141-171. 
Grossberg, S., \& Todorović, D. (1988). Neural dynamics of 1-D and 2-D brightness perception: A unified model of classical and recent phenomena. Perception \& Psychophysics, 43, 241-277.

Grove, A., Grossberg, S., \& Mingolla, E. (1995). Brightness perception, illusory contours, and corticogeniculate feedback. Visual Neuroscience, 12, 1027-1052.

Jiang, Y., \& Chun, M. M. (2001). Asymmetric object substitution masking. Journal of Experimental Psychology: Human Perception \& Performance, 27, 895-918.

KAHNEmAn, D. (1968). Methods, findings, and theory in studies of visual masking. Psychological Bulletin, 70, 404-425.

Kanizsa, G. (1976, April). Subjective contours. Scientific American, 234, 48-52.

Mack, A., Benyu, T., Tuma, R., Kahn, S., \& Rock, I. (1992). Perceptual organization and attention. Cognitive Psychology, 24, 475-501.

Moore, C. M., \& Egeth, H. (1997). Perception without attention: Evidence of grouping under conditions of inattention. Journal of Experimental Psychology: Human Perception \& Performance, 23, 339-352.

Neill, W. T., Hutchison, K. A., \& Graves, D. F. (2002). Masking by object substitution: Dissociation of masking and cuing effects. Journal of Experimental Psychology: Human Perception \& Performance, 28, 682-694.

Palmer, S. E. (1992). Common region: A new principle of perceptual grouping. Cognitive Psychology, 24, 436-447.

SCHNEIDER, W. (1988). Micro Experimental Laboratory: An integrated system for IBM PC compatibles. Behavior Research Methods, Instruments, \& Computers, 20, 206-217.

Sillito, A. M., Jones, H. E., Gerstein, G. L., \& West, D. C. (1994). Feature-linked synchronization of thalamic relay cell firing induced by feedback from the visual cortex. Nature, 369, 479-482.

YIN, R. (1969). Looking at upside-down faces. Journal of Experimental Psychology, 81, 141-145.

ZEKI, S. M. (1993). A vision of the brain. Oxford: Blackwell.

\section{NOTES}

1. We chose this nomenclature because it focuses on our procedures, rather than on the number of dots in the masking display or the theoretical mechanism that produces the effect. In our experiments, the target and the mask were always presented concurrently-hence, common onset. However, we do not always use four dots and our intent was not to test the object substitution hypothesis.

2. When set sizes are large, the briefly masked control method is used because the masking pattern serves as the cue for the target. When the target is presented singly, either method can be used. Masking for singly presented items has been reliably reported only with the unmasked control method (Enns \& Di Lollo, 1997, Experiments 1 and 2) and does not appear to be reliably obtained with the briefly masked control method (Di Lollo et al., 2000, Experiments 3-5). Perhaps the unmasked control method is more sensitive because the comparison group was never masked. When the briefly masked control method was used, the comparison group was masked (briefly).

3. It should be noted that this methodological concern does not undermine the results found in studies in which masking effects are examined with the briefly masked control method, because the target is always in the masked location. However, in experiments in which masking is examined with the unmasked control method, like those reported herein, bias explanations need to be ruled out.

Before we manipulated Gestalt grouping, we conducted a pilot study to ensure that we could reliably obtain common onset masking with these procedures. We also manipulated spatial attention: For half of the subjects, the fixation was centered on the screen (divided attention condition); for the other half, the fixation precued the location of the target (focused attention condition). This was done to ensure that we could replicate the standard effect of reduced masking in the focused attention condition. An interaction between masking and attention was found $\left[F(1,42)=41.344, M S_{\mathrm{e}}=0.002\right]$. People in the divided attention condition performed $13 \%$ worse at identifying the target surrounded by a mask, as compared with the unmasked situation; in the focused attention condition, no difference existed between masked and unmasked trials. These data indicate that our methods were adequate for producing standard common onset masking effects.

4. We thank Jim Neely for alerting us to this additional interpretation.

(Manuscript received January 31, 2001; revision accepted for publication March 15, 2002.) 